\title{
Bayesian estimation of reliability function for a changing exponential family model under different loss functions
}

\author{
P. Nasiri, N. Jafari, A. Jafari \\ Department of Statistics, Payam Noor university, I. R. of IRAN \\ Email address: \\ Pnasiri45@yahoo.com (P. Nasiri),nazli_jafari66@yahoo.com (N. Jafari), amrollahjafari@gmail. com (A. Jafari)
}

\section{To cite this article:}

P. Nasiri, N. Jafari, A. Jafari. Bayesian Estimation of Reliability Function for A Changing Exponential Family Model under Different Loss Functions. American Journal of Theoretical and Applied Statistics. Vol. 3, No. 1, 2014, pp. 25-30.

doi: $10.11648 /$ j.ajtas.20140301.14

\begin{abstract}
Absrtact: The paper deals with estimating shift point which occurs in any sequences of independent observations $\mathrm{x}_{1}$, $\mathrm{x}_{2}, \ldots, \mathrm{x}_{\mathrm{m}}, \mathrm{x}_{\mathrm{m}+1}, \ldots, \mathrm{x}_{\mathrm{n}}$ of poisson and geometric distributions. This shift point occurs in the sequence when $\mathrm{x}_{\mathrm{m}}$ i. e. $\mathrm{m}$ life data are observed. With known shift point ' $m$ ', the Bayes estimator on befor and after shift process means $\theta_{1}$ and $\theta_{2}$ are derived for symmetric and assymetric loss functions. The sensitivity analysis of Bayes estimators are carried out by simulation and numerical comparisons with R-programming. The results show the effectiveness of shift in sequences of both poisson and geometric distributions.
\end{abstract}

Keywords: Bayes Estimator, Exponential Family, Squared Error Loss Function, LinexLoss Function, General Entropy Loss Function, Precautionary Loss Function, Shift Point,

Poisson and Geometric Distributions

\section{Introduction}

In some real life applications, like medical science and physical systems manufacturing the items are often subject to abrupt shifts in the failure rate function, which are observed due to major operations or specific activities, that is may observed at some point of time instability in the sequence of life times. These situations are for the times when the shift point, $\mathrm{m}$, is known. there are many studies on shift point problem in a sequence of random variables. Hinkley (1970) studied the shift point problem and considered a sequence of independent continuous random variables.

Most authors' investigations are based on the work of Hinkley (1970). For example the shift point problem in a sequence of binomial variables is studied by Hinkley and Hinkley (1970); the shift point in a sequence of exponential and poisson variables are investigated by Worsley (1986); Haccou, Meelis and Geer (1988); Estimation of shift point in a homogeneous poisson process studied by Jandhyala and Fotopolus (1999) and Boudjelaba, MacGibbon and Sawyer (2001); Fotopolus and Jandhyala (2001). The study of homogeneous poisson process and continuous thime shift point problem in such poissonprocess has been carried out by some authors, For example use of cumulating sum

(CUSUM) control charts and exponentially weighted charts are studied by Montgomery (2001) and Wu et. al. (2004). Lim et. al. (2002), Wu and Tiau (2005) and Zhang and $\mathrm{Wu}$ (2005) considered the applications ofCUSUM control charts. Bayes estimation of unknown shift point in geometric distribution is studied by Shah and Patel (2006); Many of statisticians like Chin and Broemeling (1980), Calabria and Pulcini (1994), Zacks (1983), Pandaya and Jani (2006), Shah and Patel (2007, 2009), Chib (2998), Altessimo and Corradi (2003) and Fiteni (2004) studied the shift point Models in Bayesian framework, and Bayesian estimation of shift point in poisson Model is studied by gorakhpour university's authors (2012).

In this paper the Bayes estimator of mean parameter $\theta_{1}$ and $\theta_{2}$, for the sequences, befor and after shift point ' $\mathrm{m}$ ' of independent life times from poisson and geometric population are derived for symmetric and assymetric loss functions. squared error loss function, linex loss function, general entropy loss function, and precautionary loss function. A sensitivity analysis of these Bayes estimates has also been presented by simulation and numerical comparison study through R-programming. 


\section{Likelihood Functions}

Let $x_{1}, x_{2}, \ldots, x_{n}$ and $y_{1}, y_{2}, \ldots, y_{n}(n \geq 3)$ be the sequences of observed discrete life times. First let observation $\mathrm{x}_{1}, \quad \mathrm{x}_{2}, \quad \ldots, \quad \mathrm{x}_{\mathrm{n}}$ have come from poissondistribution, and $\mathrm{y}_{1}, \mathrm{y}_{2}, \ldots, \mathrm{y}_{\mathrm{n}}$ have come from geometric distribution with their probability mass functions(pmf) as

$$
\begin{aligned}
& p(x)=\frac{e^{-\theta} \theta^{x}}{x !} ; x=0,1, \ldots, \theta>0 \\
& p(y)=\theta(1-\theta)^{y} ; y=0,1,
\end{aligned}
$$

Let ' $\mathrm{m}$ ' is shift point in the observation which breaks the distribution in two sequences, that is for poisson model $\left(\mathrm{x}_{1}\right.$, $\left.\mathrm{x}_{2}, \ldots, \mathrm{x}_{\mathrm{m}}\right) \&\left(\mathrm{x}_{\mathrm{m}+1}, \ldots, \mathrm{x}_{\mathrm{n}}\right)$, and for geometric model $\left(\mathrm{y}_{1}\right.$, $\left.\mathrm{y}_{2}, \ldots, \mathrm{y}_{\mathrm{m}}\right) \&\left(\mathrm{y}_{\mathrm{m}+1}, \ldots, \mathrm{y}_{\mathrm{n}}\right)$.

The probability mass functions of the above sequences are

$$
\begin{aligned}
& p_{1}(x)=\frac{e^{-\theta_{1}} \theta_{1}{ }^{x}}{x !} ; x=0,1, \ldots, \theta_{1}>0 \\
& p_{2}(x)=\frac{e^{-\theta_{2} \theta_{2}}}{x !} ; x=0,1, \ldots, \theta_{2}>0
\end{aligned}
$$

and

$$
\begin{aligned}
& \mathrm{p}_{1}(\mathrm{y})=\theta_{1}\left(1-\theta_{1}\right)^{\mathrm{y}} ; \mathrm{y}=0,1, \\
& \mathrm{p}_{2}(\mathrm{y})=\theta_{2}\left(1-\theta_{2}\right)^{\mathrm{y}} ; \mathrm{y}=0,1,
\end{aligned}
$$

The likelihood function of p. m. f. 's of the sequences are

$$
\begin{aligned}
& \mathrm{L}\left(\theta_{1} \mid \underline{\mathrm{x}}\right)=\frac{\mathrm{e}^{-\mathrm{m} \lambda_{1} \lambda_{1}} \mathrm{~s}_{1 \mathrm{~m}}}{\mathrm{x}_{1} ! \ldots \mathrm{x}_{\mathrm{m}} !} \\
& \mathrm{L}\left(\theta_{2} \mid \underline{\mathrm{x}}\right)=\frac{\mathrm{e}^{-(\mathrm{n}-\mathrm{m}) \theta_{2}} \theta_{2}\left(\mathrm{~s}_{1 \mathrm{n}}-\mathrm{s}_{1 \mathrm{~m}}\right)}{\mathrm{x}_{(\mathrm{m}+1) ! \ldots \mathrm{x}_{\mathrm{n}} !}} \\
& \mathrm{g}\left(\theta_{1}, \theta_{2} ; \mathrm{m}\right)=\frac{\mathrm{b}_{1}{ }^{\mathrm{a}_{1}} \mathrm{~b}_{2}{ }^{\mathrm{a}_{2}}}{\Gamma\left(\mathrm{a}_{1}\right) \Gamma\left(\mathrm{a}_{2}\right)} \theta_{1}^{\mathrm{a}_{1}-1} \theta_{2}^{\mathrm{a}_{2}-1} \exp \left(-b_{1} \theta_{1}\right) \exp \left(-b_{2} \theta_{2}\right)
\end{aligned}
$$

Wheres $_{1 \mathrm{~m}}=\sum_{\mathrm{i}=1}^{\mathrm{m}} \mathrm{x}_{\mathrm{i}}, \mathrm{s}_{1 \mathrm{n}}-\mathrm{s}_{1 \mathrm{~m}}=\sum_{\mathrm{i}=\mathrm{m}+1}^{\mathrm{n}} \mathrm{x}_{\mathrm{i}}$ and

$$
\begin{gathered}
\mathrm{L}\left(\theta_{1} \mid \underline{\mathrm{y}}\right)=\theta_{1}^{\mathrm{m}}\left(1-\theta_{1}\right)^{\mathrm{s}_{1 \mathrm{~m}}} \\
\mathrm{~L}\left(\theta_{1} \mid \underline{\mathrm{y}}\right)=\theta_{2}^{(\mathrm{n}-\mathrm{m})}\left(1-\theta_{2}\right)^{\left(\mathrm{s}_{1 \mathrm{n}}-\mathrm{s}_{1 \mathrm{~m}}\right)}
\end{gathered}
$$

Where

$$
\mathrm{s}_{1 \mathrm{~m}}=\sum_{\mathrm{i}=1}^{\mathrm{m}} \mathrm{y}_{\mathrm{i}}, \mathrm{s}_{1 \mathrm{n}}-\mathrm{s}_{1 \mathrm{~m}}=\sum_{\mathrm{i}=\mathrm{m}+1}^{\mathrm{n}} \mathrm{y}_{\mathrm{i}}
$$

Then, the joint likelihood functions is given by

$$
\begin{aligned}
& \mathrm{L}\left(\theta_{1} \theta_{2} \mid \underline{\mathrm{x}}\right)=\frac{\mathrm{e}^{-\mathrm{m} \theta_{1} \lambda_{1}} \mathrm{~s}_{1 \mathrm{~m}}}{\mathrm{x}_{1} ! \ldots \mathrm{x}_{\mathrm{m}} !} \times \frac{\mathrm{e}^{-(\mathrm{n}-\mathrm{m}) \theta_{2} \theta_{2}}{ }^{\left(\mathrm{s}_{1 \mathrm{n}}-\mathrm{s}_{1} \mathrm{~m}\right)}}{\mathrm{x}_{(\mathrm{m}+1)} ! \ldots \mathrm{x}_{\mathrm{n}} !} \\
& \mathrm{L}\left(\theta_{1} \theta_{2} \mid \underline{\mathrm{y}}\right)=\theta_{1}{ }^{\mathrm{m}}\left(1-\theta_{1}\right)^{\mathrm{s}_{1 \mathrm{~m}}} \times \theta_{2}{ }^{(\mathrm{n}-\mathrm{m})}\left(1-\theta_{2}\right)^{\left(\mathrm{s}_{1 \mathrm{n}}-\mathrm{s}_{1 \mathrm{~m}}\right)}
\end{aligned}
$$

Suppose the marginal prior distributions of $\theta_{1}, \theta_{2}$ are natural conjugate priorfor poisson model:

$$
\begin{aligned}
& g_{1}\left(\theta_{1}\right)=\frac{b_{1}{ }^{a_{1}}}{\Gamma\left(a_{1}\right)} \theta_{1}{ }^{a_{1}-1} e^{-b_{1} \theta_{1}} ; a_{1}, b_{1}>0 \\
& g_{2}\left(\theta_{2}\right)=\frac{b_{2}{ }^{a_{2}}}{\Gamma\left(a_{2}\right)} \theta_{2}{ }^{a_{2}-1} e^{-b_{2} \theta_{2}} ; a_{2}, b_{2}>0
\end{aligned}
$$

and for geometric Model:

$$
\begin{aligned}
& \mathrm{g}_{1}\left(\theta_{1}\right)=\frac{\Gamma\left(\mathrm{a}_{1}+\mathrm{b}_{1}\right)}{\Gamma\left(\mathrm{a}_{1}\right) \Gamma\left(\mathrm{b}_{1}\right)}\left(\theta_{1}\right)^{\mathrm{a}_{1}-1}\left(1-\theta_{1}\right)^{\mathrm{b}_{1}-1} ; \mathrm{a}_{1}, b_{1}>0 \\
& \mathrm{~g}_{2}\left(\theta_{2}\right)=\frac{\Gamma\left(\mathrm{a}_{2}+\mathrm{b}_{2}\right)}{\Gamma\left(\mathrm{a}_{2}\right) \Gamma\left(\mathrm{b}_{2}\right)}\left(\theta_{2}\right)^{\mathrm{a}_{2}-1}\left(1-\theta_{2}\right)^{\mathrm{b}_{2}-1} ; \mathrm{a}_{2}, \mathrm{~b}_{2}>0
\end{aligned}
$$

The joint prior distribution of $\theta_{1}, \theta_{2}$ and shift point 'm', according to Ganji(2010) by using the exponential family

In case of, geometric model:

$$
\mathrm{g}\left(\theta_{1}, \theta_{2} ; \mathrm{m}\right)=\frac{\Gamma\left(\mathrm{a}_{1}+\mathrm{b}_{1}\right)}{\Gamma\left(\mathrm{a}_{1}\right) \Gamma\left(\mathrm{b}_{1}\right)} \times \frac{\Gamma\left(\mathrm{a}_{2}+\mathrm{b}_{2}\right)}{\Gamma\left(\mathrm{a}_{2}\right) \Gamma\left(\mathrm{b}_{2}\right)}\left(\theta_{1}\right)^{\mathrm{a}_{1}-1}\left(1-\theta_{1}\right)^{\mathrm{b}_{1}-1}\left(\theta_{2}\right)^{\mathrm{a}_{2}-1}\left(1-\theta_{2}\right)^{\mathrm{b}_{2}-1}
$$

where $\theta_{1}, \theta_{2}>0$ and $\mathrm{m}=1, \ldots,(\mathrm{n}-1)$

The joint posterior density of $\theta_{1}, \theta_{2}$ and $\mathrm{m}$ is obtained by using equations (12), (18) for poissonmodel, and (13), (19) for geometric Model

$$
\mathrm{p}\left(\theta_{1}, \theta_{2} ; \mathrm{m} \mid \underline{\mathrm{x}}\right)=\frac{\mathrm{e}^{-\left(\mathrm{b}_{1}+\mathrm{m}\right) \theta_{1} \theta_{1}\left(\mathrm{a}_{1}+\mathrm{s}_{1 \mathrm{~m}}-1\right)} \mathrm{e}^{-\left(\mathrm{b}_{2}+\mathrm{n}-\mathrm{m}\right) \theta_{2} \theta_{2}\left(\mathrm{a}_{2}+\mathrm{s}_{1 \mathrm{n}}-\mathrm{s}_{1 \mathrm{~m}}-1\right)}}{\mathrm{D}\left(\mathrm{a}_{1}, \mathrm{a}_{2}, \mathrm{~b}_{1}, \mathrm{~b}_{2}, \mathrm{~m}, \mathrm{n}\right)}
$$

where

$$
\begin{aligned}
& D\left(a_{1}, a_{2}, b_{1}, b_{2}, m, n\right)=\left[\frac{\Gamma\left(a_{1}+s_{1 m}\right)}{\left(b_{1}+m\right)^{\left(a_{1}+s_{1 m}\right)}} \times \frac{\Gamma\left(a_{2}+s_{1 n}-s_{1 m}\right)}{\left(b_{2}+n-m\right)^{\left(a_{2}+s_{1 n}-s_{1 m}\right)}}\right] \\
& p\left(\theta_{1}, \theta_{2} ; m \mid \underline{y}\right)=\frac{\left(\theta_{1}\right)^{\left(a_{1}+m-1\right)}\left(1-\theta_{1}\right)^{\left(b_{1}+s_{1 m}-1\right)}\left(\theta_{2}\right)^{\left(a_{2}+n-m-1\right)}\left(1-\theta_{2}\right)^{\left(b_{2}+s_{1} n^{-} s_{1}-1\right)}}{D\left(a_{1}, a_{2}, b_{1}, b_{2}, m, n\right)}
\end{aligned}
$$

where

$$
\mathrm{D}\left(\mathrm{a}_{1}, \mathrm{a}_{2}, \mathrm{~b}_{1}, \mathrm{~b}_{2}, \mathrm{~m}, \mathrm{n}\right)=\left[\frac{\Gamma\left(\mathrm{a}_{1}+\mathrm{m}\right) \Gamma\left(\mathrm{b}_{1}+\mathrm{s}_{1 \mathrm{~m}}\right)}{\Gamma\left(\mathrm{a}_{1}+\mathrm{m}+\mathrm{b}_{1}+\mathrm{s}_{1 \mathrm{~m}}\right)} \times \frac{\Gamma\left(\mathrm{a}_{2}+\mathrm{n}-\mathrm{m}\right) \Gamma\left(\mathrm{b}_{2}+\mathrm{s}_{1 \mathrm{n}}-\mathrm{s}_{1 \mathrm{~m}}\right)}{\Gamma\left(\mathrm{a}_{2}+\mathrm{n}-\mathrm{m}+\mathrm{b}_{2}+\mathrm{s}_{1 \mathrm{n}}-\mathrm{s}_{1 \mathrm{~m}}\right)}\right]
$$


The marginal posterior distribution of $\theta_{1}$, forpoissonmodel, by using the equations (12), (14) and dor geometric Model, by using the equations (13), (16)

$$
\begin{aligned}
& \mathrm{p}\left(\theta_{1} \mid \underline{\mathrm{x}}\right)=\frac{\left[\mathrm{e}^{-\left(\mathrm{b}_{1}+\mathrm{m}\right) \theta_{1} \theta_{1}\left(\mathrm{a}_{1}+\mathrm{s}_{1} \mathrm{~m}^{-1)}\right.} \frac{\Gamma\left(\mathrm{a}_{2}+\mathrm{s}_{1 \mathrm{n}}-\mathrm{s}_{1 \mathrm{~m}}\right)}{\left.\left(\mathrm{b}_{2}+\mathrm{n}-\mathrm{m}\right)^{\left(\mathrm{a}_{2}+\mathrm{s}_{1} \mathrm{~s}^{-s_{1 m}}\right)}\right]}\right.}{\mathrm{D}\left(\mathrm{a}_{1}, \mathrm{a}_{2}, \mathrm{~b}_{1}, \mathrm{~b}_{2}, \mathrm{~m}, \mathrm{n}\right)} \\
& \mathrm{p}\left(\theta_{1} \mid \underline{\mathrm{y}}\right)=\frac{\left(\theta_{1}\right)^{\left(\mathrm{a}_{1}+\mathrm{m}-1\right)}\left(1-\theta_{1}\right)^{\left(\mathrm{b}_{1}+\mathrm{s}_{1}-1\right)} \times \frac{\Gamma\left(\mathrm{a}_{2}+\mathrm{n}-\mathrm{m}\right) \Gamma\left(\mathrm{b}_{2}+\mathrm{s}_{1} \mathrm{n}^{-s_{1}}\right)}{\Gamma\left(\mathrm{a}_{2}+\mathrm{n}-\mathrm{m}+\mathrm{b}_{2}+\mathrm{s}_{1} \mathrm{n}^{-} \mathrm{s}_{1 \mathrm{~m}}\right)}}{\mathrm{D}\left(\mathrm{a}_{1}, \mathrm{a}_{2}, \mathrm{~b}_{1}, \mathrm{~b}_{2}, \mathrm{~m}, \mathrm{n}\right)}
\end{aligned}
$$

the marginal posterior distribution of $\theta_{2}$, for poissonModel, by using the equations (12), (15) and for geometric Model, by using the equations (13), (17)

$$
\begin{aligned}
& \mathrm{p}\left(\theta_{2} \mid \underline{\mathrm{x}}\right)=\frac{\mathrm{e}^{-\left(\mathrm{b}_{2}+\mathrm{n}-\mathrm{m}\right) \theta_{2} \theta_{2}\left(\mathrm{a}_{2}+\mathrm{s}_{1} \mathrm{n}^{\left.-\mathrm{s}_{1}-1\right)} \frac{\Gamma\left(\mathrm{a}_{1}+\mathrm{s}_{1 \mathrm{~m}}\right)}{\left(\mathrm{b}_{1}+\mathrm{m}\right)^{\left(\mathrm{a}_{1}+\mathrm{s}_{1} \mathrm{~m}\right)}}\right.}}{\mathrm{D}\left(\mathrm{a}_{1}, \mathrm{a}_{2}, \mathrm{~b}_{1}, \mathrm{~b}_{2}, \mathrm{~m}, \mathrm{n}\right)} \\
& \mathrm{p}\left(\theta_{2} \mid \underline{\mathrm{y}}\right)=\frac{\left(\theta_{2}\right)^{\left(\mathrm{a}_{2}+\mathrm{n}-\mathrm{m}-1\right)}\left(1-\theta_{2}\right)^{\left(\mathrm{b}_{2}+\mathrm{s}_{1} \mathrm{n}^{\left.-s_{1}-1\right)} \times \frac{\Gamma\left(\mathrm{a}_{1}+\mathrm{m}\right) \Gamma\left(\mathrm{b}_{1}+\mathrm{s}_{1 \mathrm{~m}}\right)}{\Gamma\left(\mathrm{a}_{1}+\mathrm{m}+\mathrm{b}_{1}+\mathrm{s}_{1}\right)}\right.}}{\mathrm{D}\left(\mathrm{a}_{1}, \mathrm{a}_{2}, \mathrm{~b}_{1}, \mathrm{~b}_{2}, \mathrm{~m}, \mathrm{n}\right)}
\end{aligned}
$$

\section{Bayes Estimators under Symmetric and Asymmetric Loss Functions}

In decision theory the loss criterion is specified in order to obtain best estimator. The simplest form of loss function is squared error loss function (SELF) which assigns equal magnitudes to both positive and negative errors. However this assumption may be inappropriate in most of the estimation problems. some time overestimation leads to many serious qonsequences. In such situation many outhors found the asymmetric loss functions, moreappropriate. In this paper we have considered some of the asymmetric loss functions, Linex loss function (LLF) suggested and studied by Varian (1975), Zellner (1986) , Basu and Ebrahimi (1991), General entropy loss function (GELF) by Calabria and Pulcini (1996) and Precautionary loss function (PLF) studied by Norstrom (1996). Such asymmetric loss functions are also studied by Ohtani (1995), Parsian and Kirmani (2002), Brases and Dette (2004), Pandyaet. al. (2004) and Gorakhpour university's authors (2012).

\subsection{Bayes Estimators under Squared Error Loss Functions (SELF)}

From a decision theorical view point, in order to select value as representing on 'best' estimator, a loss function must be specified, In this section we consider SELF.

The Bayes estimate of a generic parameter $\theta$ based on a SELF is given by $L_{1}(\theta, d)=(\theta-d)^{2}$, where ' $d$ ' is a decision rule to estimate $\theta$, is posterior mean.

The Bayes estimate $\hat{\theta}_{1 B S}$ of $\theta_{1}$ under SELF using marginal posterior density equation (24) for poisson model is given by

$$
\begin{aligned}
\hat{\theta}_{1 B S} & \left.=\frac{\left[\frac{\Gamma\left(a_{1}+s_{1 m}+1\right)}{\left(b_{1}+m\right)^{\left(a_{1}+s_{1} m+1\right)}} \times \frac{\Gamma\left(a_{2}+s_{1 n}-s_{1 m}\right)}{\left(b_{2}+n-m\right)}\right]}{D\left(a_{1}, a_{2}, b_{1}, b_{2}, m, n\right)}\right] \\
\hat{\theta}_{1 B S} & =\frac{\left.D\left(a_{1}+1\right), a_{2}, b_{1}, b_{2}, m, n\right)}{D\left(a_{1}, a_{2}, b_{1}, b_{2}, m, n\right)}
\end{aligned}
$$

and equation (25) for geometric model is given by

$$
\begin{aligned}
& \hat{\theta}_{1 \mathrm{BS}}=\frac{\left[\frac{\Gamma\left(\mathrm{a}_{1}+\mathrm{m}+1\right) \Gamma\left(\mathrm{b}_{1}+\mathrm{s}_{1 \mathrm{~m}}\right)}{\Gamma\left(\mathrm{a}_{1}+\mathrm{m}+\mathrm{b}_{1}+\mathrm{s}_{1} \mathrm{~m}+1\right)} \times \frac{\Gamma\left(\mathrm{a}_{2}+\mathrm{n}-\mathrm{m}\right) \Gamma\left(\mathrm{b}_{2}+\mathrm{s}_{1 \mathrm{n}}-\mathrm{s}_{1 \mathrm{~m}}\right)}{\Gamma\left(\mathrm{a}_{2}+\mathrm{n}-\mathrm{m}+\mathrm{b}_{2}+\mathrm{s}_{1} \mathrm{n}^{-s_{1}}\right)}\right]}{\mathrm{D}\left(\mathrm{a}_{1}, \mathrm{a}_{2}, \mathrm{~b}_{1}, \mathrm{~b}_{2}, \mathrm{~m}, \mathrm{n}\right)} \\
& \hat{\theta}_{1 \mathrm{BS}}=\frac{\mathrm{D}\left(\left(\mathrm{a}_{1}+1\right), \mathrm{a}_{2}, \mathrm{~b}_{1}, \mathrm{~b}_{2}, \mathrm{~m}, \mathrm{n}\right)}{\mathrm{D}\left(\mathrm{a}_{1}, \mathrm{a}_{2}, \mathrm{~b}_{1}, \mathrm{~b}_{2}, \mathrm{~m}, \mathrm{n}\right)}
\end{aligned}
$$

The Bayes estimate $\hat{\theta}_{2 B S}$ of $\theta_{2}$ under SELF using marginal posterior density equation (26) for poisson model is given by

$$
\begin{aligned}
\hat{\theta}_{2 B S} & =\frac{\left[\frac{\Gamma\left(a_{2}+s_{1 n}-s_{1 m}+1\right)}{\left(b_{2}+n-m\right)^{\left(a_{2}+s_{1 n}-s_{1 m}+1\right)}} \times \frac{\Gamma\left(a_{1}+s_{1 m}\right)}{\left(b_{1}+m\right)^{\left(a_{1}+s_{1 m}\right)}}\right]}{D\left(a_{1}, a_{2}, b_{1}, b_{2}, m, n\right)} \\
\hat{\theta}_{2 B S} & =\frac{D\left(a_{1},\left(a_{2}+1\right), b_{1}, b_{2}, m, n\right)}{D\left(a_{1}, a_{2}, b_{1}, b_{2}, m, n\right)}
\end{aligned}
$$

The equation (27) for geometric model is given by

$$
\begin{aligned}
& \hat{\theta}_{2 B S}=\frac{\left[\frac{\Gamma\left(a_{1}+m\right) \Gamma\left(b_{1}+s_{1}\right)}{\Gamma\left(a_{1}+m+b_{1}+s_{1 m}\right)} \times \frac{\Gamma\left(a_{2}+n-m+1\right) \Gamma\left(b_{2}+s_{1}-s_{1 m}\right)}{\Gamma\left(a_{2}+n-m+b_{2}+s_{1} n^{-} s_{1}+1\right)}\right]}{D\left(a_{1}, a_{2}, b_{1}, b_{2}, m, n\right)} \\
& \hat{\theta}_{2 B S}=\frac{D\left(a_{1},\left(a_{2}+1\right), b_{1}, b_{2}, m, n\right)}{D\left(a_{1}, a_{2}, b_{1}, b_{2}, m, n\right)}
\end{aligned}
$$

\subsection{Bayes Estimator under Linex Loss function (LLF)}

The asymmetric loss function given by Varian (1975). known as Linex loss function (LLF), is defined by

$$
L_{2}(\theta, d)=\exp \left[\gamma_{1}(d-\theta)\right]-\gamma_{1}(d-\theta)-1 ; \gamma_{1} \neq 0
$$

Where $\mathrm{d}$ is the decision rule to estimate unknown parameter $\theta$.

The Bayes estimate $\hat{\theta}_{1 B L}$ of $\theta_{1}$ using marginal posterior density equation (24) under LLF, forpoisson model is given by

$$
\begin{aligned}
& \hat{\theta}_{1 \mathrm{BL}}=\frac{-1}{\gamma_{1}} \ln \left[\frac{\left[\frac{\Gamma\left(\mathrm{a}_{1}+\mathrm{s}_{1 \mathrm{~m}}+1\right)}{\left(\mathrm{b}_{1}+\mathrm{m}+\gamma_{1}\right)^{\left(a_{1}+s_{1}+1\right)}} \times \frac{\Gamma\left(\mathrm{a}_{2}+\mathrm{s}_{1} \mathrm{n}^{-} \mathrm{s}_{1 \mathrm{~m}}\right)}{\left.\left(\mathrm{b}_{2}+\mathrm{n}-\mathrm{m}\right)^{\left(\mathrm{a}_{2}+\mathrm{s}_{1} \mathrm{n}^{-} \mathrm{s}_{1 \mathrm{~m}}\right)}\right]}\right.}{\mathrm{D}\left(\mathrm{a}_{1}, \mathrm{a}_{2}, \mathrm{~b}_{1}, \mathrm{~b}_{2}, \mathrm{~m}, \mathrm{n}\right)}\right] \\
& \hat{\theta}_{1 \mathrm{BL}}=\frac{-1}{\gamma_{1}} \ln \left[\frac{\mathrm{D}\left(\mathrm{a}_{1}, \mathrm{a}_{2},\left(\mathrm{~b}_{1}+\gamma_{1}\right), \mathrm{b}_{2}, \mathrm{~m}, \mathrm{n}\right)}{\mathrm{D}\left(\mathrm{a}_{1}, \mathrm{a}_{2}, \mathrm{~b}_{1}, \mathrm{~b}_{2}, \mathrm{~m}, \mathrm{n}\right)}\right]
\end{aligned}
$$

The Bayes estimate of $\hat{\theta}_{2 \mathrm{BL}}$ of $\theta_{2}$ using marginal 
posterior density equation (26) under LLF , forpoisson model is given by

$$
\begin{aligned}
& \hat{\theta}_{2 B L}=\frac{-1}{\gamma_{1}} \ln \left[\frac{\left[\frac{\Gamma\left(a_{1}+s_{1 m}\right)}{\left(b_{1}+m\right)^{\left(a_{1}+s_{1 m}\right)}} \times \frac{\Gamma\left(a_{2}+s_{1 n}-s_{1 m}\right)}{\left(b_{2}+n-m+\gamma_{1}\right)^{\left(a_{2}+s_{1 n}-s_{1 m}\right)}}\right]}{D\left(a_{1}, a_{2}, b_{1}, b_{2}, m, n\right)}\right] \\
& \hat{\theta}_{2 B L}=\frac{-1}{\gamma_{1}} \ln \left[\frac{\mathrm{D}\left(a_{1}, a_{2}, b_{1},\left(b_{2}+\gamma_{1}\right), m, n\right)}{D\left(a_{1}, a_{2}, b_{1}, b_{2}, m, n\right)}\right]
\end{aligned}
$$

We can not use linex loss function for geometric loss function.

\subsection{Bayes Estimator under General Entropy Loss Function}

Occasionally, the use of symmetric loss function, namely SELF, was found inappropriate, since for example, an overestimation of reliability function usually much more serious than an underestimation. Here was considered asymmetric loss function namely general entropy loss function (GELF) proposed by Calabria and Pulcini (1994), is given by

$$
\mathrm{L}_{3}(\theta, \mathrm{d})=\left(\frac{\mathrm{d}}{\theta}\right)^{\gamma_{2}}-\gamma_{2} \ln \left(\frac{\mathrm{d}}{\theta}\right)-1 ; \gamma_{2} \neq 0
$$

The Bayes estimate $\hat{\theta}_{1 \mathrm{BE}}$ of $\theta_{1}$, unde GELF using marginal posterior distribution equation (24) for poissonmodel, is given by

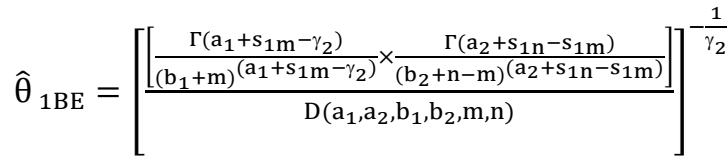

$$
\begin{aligned}
& \hat{\theta}_{1 \mathrm{BE}}=\left[\frac{\mathrm{D}\left(\left(\mathrm{a}_{1}-\gamma_{2}\right), \mathrm{a}_{2}, \mathrm{~b}_{1}, \mathrm{~b}_{2}, \mathrm{~m}, \mathrm{n}\right)}{\mathrm{D}\left(\mathrm{a}_{1}, \mathrm{a}_{2}, \mathrm{~b}_{1}, \mathrm{~b}_{2}, \mathrm{~m}, \mathrm{n}\right)}\right]^{-\frac{1}{\gamma_{2}}}
\end{aligned}
$$

and equation (25) for geometric model is given by

$$
\begin{aligned}
& \hat{\theta}_{1 \mathrm{BE}}=\left[\frac{\left[\frac{\Gamma\left(\mathrm{a}_{1}+\mathrm{m}+\gamma_{2}\right) \Gamma\left(\mathrm{b}_{1}+\mathrm{s}_{1 \mathrm{~m}}\right)}{\Gamma\left(\mathrm{a}_{1}+\mathrm{m}+\gamma_{2}+\mathrm{b}_{1}+\mathrm{s}_{1 \mathrm{~m}}\right)} \times \frac{\Gamma\left(\mathrm{a}_{2}+\mathrm{n}-\mathrm{m}\right) \Gamma\left(\mathrm{b}_{2}+\mathrm{s}_{1 \mathrm{n}}-\mathrm{s}_{1 \mathrm{~m}}\right)}{\Gamma\left(\mathrm{a}_{2}+\mathrm{n}-\mathrm{m}+\mathrm{b}_{2}+\mathrm{s}_{1 \mathrm{n}}-\mathrm{s}_{1 \mathrm{~m}}\right)}\right]}{\mathrm{D}\left(\mathrm{a}_{1}, \mathrm{a}_{2}, \mathrm{~b}_{1}, \mathrm{~b}_{2}, \mathrm{~m}, \mathrm{n}\right)}\right]^{\frac{-1}{\gamma_{2}}} \\
& \hat{\theta}_{1 \mathrm{BE}}=\left[\frac{\mathrm{D}\left(\left(\mathrm{a}_{1}+\gamma_{2}\right), \mathrm{a}_{2}, \mathrm{~b}_{1}, \mathrm{~b}_{2}, \mathrm{~m}, \mathrm{n}\right)}{\mathrm{D}\left(\mathrm{a}_{1}, \mathrm{z}_{2}, \mathrm{~b}_{1}, \mathrm{~b}_{2}, \mathrm{~m}, \mathrm{n}\right)}\right]^{\frac{-1}{\gamma_{2}}}
\end{aligned}
$$

The Bayes estimate $\hat{\theta}_{2 \mathrm{BE}}$ of $\theta_{2}$, unde GELF using marginal posterior distribution equation (26) for poissonmodel, is given by

$$
\begin{aligned}
& \hat{\theta}_{2 B E}=\left[\frac{\left.\frac{\Gamma\left(a_{1}+s_{1 m}\right)}{\left(b_{1}+m\right)^{\left(a_{1}+s_{1 m}\right)}} \times \frac{\Gamma\left(a_{2}+s_{1 n}-s_{1 m}-\gamma_{2}\right)}{D\left(a_{1}, a_{2}, b_{1}, b_{2}, m, n\right)}\right]^{\left(a_{2}+s_{1 n}-s_{1 m}-\gamma_{2}\right)}}{-\frac{1}{\gamma_{2}}}\right]^{-a_{2 B E}}=\left[\frac{D\left(a_{1},\left(a_{2}-\gamma_{2}\right), b_{1}, b_{2}, m, n\right)}{D\left(a_{1}, a_{2}, b_{1}, b_{2}, m, n\right)}\right]^{-\frac{1}{\gamma_{2}}} \\
& \hat{\theta}_{2}
\end{aligned}
$$

and equation (27) for geometric model is given by

$$
\begin{aligned}
& \hat{\theta}_{2 B E}=\left[\frac{\left[\frac{\Gamma\left(a_{1}+m\right) \Gamma\left(b_{1}+s_{1 m}\right)}{\Gamma\left(a_{1}+m+b_{1}+s_{1 m}\right)} \times \frac{\Gamma\left(a_{2}+n-m-\gamma_{2}\right) \Gamma\left(b_{2}+s_{1 n}-s_{1 m}\right)}{\Gamma\left(a_{2}+n-m-\gamma_{2}+b_{2}+s_{1 n}-s_{1}\right)}\right]}{D\left(a_{1}, a_{2}, b_{1}, b_{2}, m, n\right)}\right]^{\frac{-1}{\gamma_{2}}} \\
& \hat{\theta}_{2 B E}=\left[\frac{D\left(a_{1},\left(a_{2}-\gamma_{2}\right), b_{1}, b_{2}, m, n\right)}{D\left(a_{1}, a_{2}, b_{1}, b_{2}, m, n\right)}\right]^{\frac{-1}{\gamma_{2}}}
\end{aligned}
$$

\subsection{Bayes Estimators under Precautioanary Loss Function (PLF)}

Norstrom (1996) introduced an alternative asymmetric loss function and also presented a general class of precautionary loss function with quadratic loss function as a special case. These loss functions approach infinitely near the origin to prevent the overestimation and thus giving conservative estimators, especially when low failure rates are being estimated which may lead to serious consequences.

A very useful and simple asymmetric precautionary loss function is given by

$$
\mathrm{L}_{4}(\theta, \mathrm{d})=\frac{(\theta-\mathrm{d})^{2}}{\mathrm{~d}}
$$

The Bayes estimator $\hat{\theta}_{1 \mathrm{BP}}$ of $\theta_{1}$, under PLF using the marginal posterior distribution (24) for poisson model is given by

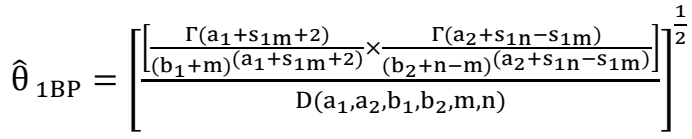

$$
\begin{aligned}
& \hat{\theta}_{1 B P}=\left[\frac{D\left(\left(a_{1}+2\right), a_{2}, b_{1}, b_{2}, m, n\right)}{D\left(a_{1}, a_{2}, b_{1}, b_{2}, m, n\right)}\right]^{\frac{1}{2}}
\end{aligned}
$$

and equation (25) for geometric model is given by

$$
\begin{aligned}
& \hat{\theta}_{1 B P}=\left[\frac{\left[\frac{\Gamma\left(a_{1}+m+2\right) \Gamma\left(b_{1}+s_{1 m}\right)}{\Gamma\left(a_{1}+m+b_{1}+s_{1 m}+2\right)} \times \frac{\Gamma\left(a_{2}+n-m\right) \Gamma\left(b_{2}+s_{1 n}-s_{1 m}\right)}{\Gamma\left(a_{2}+n-m+b_{2}+s_{1 n}-s_{1 m}\right)}\right]}{D\left(a_{1}, a_{2}, b_{1}, b_{2}, m, n\right)}\right] \\
& \hat{\theta}_{1 B P}=\left[\frac{D\left(\left(a_{1}+2\right), a_{2}, b_{1}, b_{2}, m, n\right)}{D\left(a_{1}, a_{2}, b_{1}, b_{2}, m, n\right)}\right]^{\frac{1}{2}}
\end{aligned}
$$

The Bayes estimator $\hat{\theta}_{2 \mathrm{BP}}$ of $\theta_{2}$, under PLF using the marginal posterior distribution (26) for poisson model is given by

$$
\begin{aligned}
& \left.\hat{\theta}_{2 B P}=\left[\frac{\left[\frac{\Gamma\left(a_{1}+s_{1 m}\right)}{\left(b_{1}+m\right)\left(a_{1}+s_{1 m}\right)} \times \frac{\Gamma\left(a_{2}+s_{1 n}-s_{1 m}+2\right)}{\left(a_{2}, b_{2}-n-m\right)\left(a_{2}+s_{1 n}-s_{1 m}+2\right)}\right.}{D\left(a_{1}, a_{2}, b_{1}, b_{2}, m, n\right)}\right]^{\frac{1}{2}}\right]^{\frac{1}{2}} \\
& \hat{\theta}_{2 B P}=\left[\frac{D\left(a_{1},\left(a_{2}+2\right), b_{1}, b_{2}, m, n\right)}{D\left(a_{1}, a_{2}, b_{1}, b_{2}, m, n\right)}\right]^{\frac{1}{2}}
\end{aligned}
$$

and equation (27) for geometric model is given by

$$
\begin{aligned}
& \hat{\theta}_{2 B P}=\left[\frac{\left[\frac{\Gamma\left(a_{1}+m\right) \Gamma\left(b_{1}+s_{1} m\right)}{\Gamma\left(a_{1}+m+b_{1}+s_{1 m}\right)} \times \frac{\Gamma\left(a_{2}+n-m+2\right) \Gamma\left(b_{2}+s_{1 n}-s_{1 m}\right)}{\Gamma\left(a_{2}+n-m+b_{2}+s_{1 n}-s_{1 m}+2\right)}\right]}{D\left(a_{1}, a_{2}, b_{1}, b_{2}, m, n\right)}\right]^{\frac{1}{2}} \\
& \hat{\theta}_{2 B P}=\left[\frac{D\left(a_{1},\left(a_{2}+2\right), b_{1}, b_{2}, m, n\right)}{D\left(a_{1}, a_{2}, b_{1}, b_{2}, m, n\right)}\right]^{\frac{1}{2}}
\end{aligned}
$$




\subsection{Numerical Comparison}

We have generated the random samples of different sizes 10 , $15,20,25,30,35,40,45,50$ with known shift points $5,10,15$, $20,25,30,35,40,45$ repectively for each of poisson and geometric distributions. We also get $\theta=2$ in poissonmodel, and $\theta=0.2$ in geometric model. The Bayes estimators of $\theta_{1}$ and $\theta_{2}$ for each distributions are calculated under Squared Error Loss Function, Linex Loss Function, General Entropy Loss Function and Precautionary Loss function by making programs in R-2. 13. 2 statistical software.

\subsection{Sensitivity Analysis of Bayes Estimation}

In this section we have studied the sensitivity of the Bayes estimators of $\theta_{1}, \theta_{2}$ whth respect to the parameters of prior distribution $a_{1}, a_{2}, b_{1}$ and $b_{2}$. We have computed the Bayes estimators of $\theta_{1}$ and $\theta_{2}$ under SELF, LLF, GELF and PLF. we have also considered different sample sizes $\mathrm{n}=10(05) 50$.

Table 1. shows the Bayes estimarors of $\theta_{1}$ and $\theta_{2}$ under SELF, LLF, GELF, PLF, and Loss functions of them that $L_{1}=L\left(\hat{\theta}_{1 B S}, \theta\right), L_{2}=L\left(\hat{\theta}_{2 B S}, \theta\right)$, $L_{3}=L\left(\hat{\theta}_{1 B L}, \theta\right), L_{4}=L\left(\hat{\theta}_{2 B L}, \theta\right), L_{5}=L\left(\hat{\theta}_{1 B E}, \theta\right), L_{6}=L\left(\hat{\theta}_{2 B E}, \theta\right), L_{7}=L\left(\hat{\theta}_{1 B P}, \theta\right), L_{8}=L\left(\hat{\theta}_{2 B P}, \theta\right)$.

\begin{tabular}{|c|c|c|c|c|c|c|c|c|c|}
\hline $\mathrm{n}$ & $\mathbf{m}$ & $\widehat{\theta}_{1 B S}$ & $\widehat{\boldsymbol{\theta}}_{2 B S}$ & $\widehat{\theta}_{1 B L}$ & $\widehat{\boldsymbol{\theta}}_{2 B L}$ & $\widehat{\theta}_{1 B E}$ & $\widehat{\boldsymbol{\theta}}_{2 B E}$ & $\widehat{\theta}_{1 B P}$ & $\widehat{\boldsymbol{\theta}}_{2 B P}$ \\
\hline 10 & 5 & 2.00 & 1.83 & 1.57 & 1. 61 & 1.78 & 1. 61 & 2.07 & 1.89 \\
\hline 15 & 10 & 2. 08 & 1. 11 & 1.92 & 0.98 & 1.96 & 0.89 & 2. 12 & 1. 83 \\
\hline 20 & 15 & 3.07 & 1. 11 & 2. 09 & 1. 23 & 2.98 & 1. 18 & 3. 10 & 1. 47 \\
\hline 25 & 20 & 2. 09 & 1.97 & 2. 00 & 1. 73 & 2. 02 & 1. 75 & 2. 11 & 2. 04 \\
\hline 30 & 25 & 1.85 & 0.68 & 1. 78 & 0.60 & 1. 79 & 0.46 & 1. 87 & 0.75 \\
\hline 35 & 30 & 2. 22 & 1. 83 & 2. 15 & 1. 61 & 2. 17 & 1. 61 & 2. 24 & 1. 89 \\
\hline 40 & 35 & 1.89 & 1. 40 & 1.84 & 1. 23 & 1. 85 & 1. 18 & 1.90 & 1. 46 \\
\hline 45 & 40 & 1. 93 & 1. 54 & 1. 88 & 1. 36 & 1.89 & 1. 33 & 1. 94 & 1. 61 \\
\hline 50 & 45 & 2. 00 & 1. 54 & 1.96 & 1. 36 & 1.99 & 1.33 & 2. 01 & 1. 61 \\
\hline $\mathbf{n}$ & $\mathbf{m}$ & $\mathbf{L}_{1}$ & $\mathbf{L}_{2}$ & $\mathbf{L}_{3}$ & $\mathbf{L}_{4}$ & $\mathbf{L}_{5}$ & $\mathbf{L}_{6}$ & $\mathbf{L}_{7}$ & $\mathbf{L}_{8}$ \\
\hline 10 & 5 & 0.000 & 0.029 & 0.105 & 0.240 & 0.026 & 0.081 & 0.003 & 0.005 \\
\hline 15 & 10 & 0.007 & 0.784 & 0.011 & 1. 169 & 0.001 & 0.804 & 0.007 & 0.563 \\
\hline 20 & 15 & 1. 155 & 0.784 & 3. 295 & 0.752 & 0.426 & 0.399 & 0.393 & 0.191 \\
\hline 25 & 20 & 0.008 & 0.001 & 0.000 & 0.119 & 0.000 & 0.003 & 0.006 & 0.001 \\
\hline 30 & 25 & 0.022 & 1.727 & 0.081 & 1. 855 & 0.022 & 1.968 & 0.009 & 2. 060 \\
\hline 35 & 30 & 0.048 & 0.029 & 0.052 & 0.240 & 0.014 & 0.081 & 0.024 & 0.005 \\
\hline 40 & 35 & 0.012 & 0.360 & 0.045 & 0.752 & 0.011 & 0.399 & 0.005 & 0. 191 \\
\hline 45 & 40 & 0.005 & 0.209 & 0.025 & 0.562 & 0.006 & 0.261 & 0.002 & 0.093 \\
\hline 50 & 45 & 0.000 & 0.209 & 0.003 & 0.562 & 0.000 & 0.261 & 0.000 & 0.093 \\
\hline
\end{tabular}

Table 2, shows the Bayes estimarors of $\theta_{1}$ and $\theta_{2}$ under SELF, LLF, GELF, PLF, and Loss functions of them that $L_{1}=L\left(\hat{\theta}_{1 B S}, \theta\right), L_{2}=L\left(\hat{\theta}_{2 B S}, \theta\right)$, $L_{3}=L\left(\hat{\theta}_{1 B L}, \theta\right), L_{4}=L\left(\hat{\theta}_{2 B L}, \theta\right), L_{5}=L\left(\hat{\theta}_{1 B E}, \theta\right), L_{6}=L\left(\hat{\theta}_{2 B E}, \theta\right), L_{7}=L\left(\hat{\theta}_{1 B P}, \theta\right), L_{8}=L\left(\hat{\theta}_{2 B P}, \theta\right)$.

\begin{tabular}{|c|c|c|c|c|c|c|c|}
\hline$n$ & $\mathbf{m}$ & $\widehat{\boldsymbol{\theta}}_{1 B S}$ & $\widehat{\boldsymbol{\theta}}_{2 B S}$ & $\widehat{\boldsymbol{\theta}}_{1 B E}$ & $\widehat{\boldsymbol{\theta}}_{2 B E}$ & $\widehat{\theta}_{1 B P}$ & $\widehat{\theta}_{2 B P}$ \\
\hline 10 & 5 & 0.21 & 0.13 & 0.17 & 0.10 & 0.22 & 0.14 \\
\hline 15 & 10 & 0.26 & 0.21 & 0.23 & 0.17 & 0.24 & 0.23 \\
\hline 20 & 15 & 0.43 & 0.31 & 0.41 & 0.26 & 0.44 & 0.33 \\
\hline 25 & 20 & 0.17 & 0.38 & 0.16 & 0.32 & 0.17 & 0.39 \\
\hline 30 & 25 & 0.23 & 0.36 & 0.22 & 0.31 & 0.23 & 0.38 \\
\hline 35 & 30 & 0.19 & 0.21 & 0.19 & 0.17 & 0.19 & 0.22 \\
\hline 40 & 35 & 0.23 & 0.26 & 0.22 & 0.21 & 0.23 & 0.28 \\
\hline 45 & 40 & 0.26 & 0.24 & 0.25 & 0.13 & 0.26 & 0.25 \\
\hline 50 & 45 & 0.28 & 0.22 & 0.27 & 0.18 & 0.28 & 0.23 \\
\hline n & $\mathbf{m}$ & $\mathbf{L}_{1}$ & $\mathbf{L}_{2}$ & $\mathbf{L}_{3}$ & $\mathbf{L}_{4}$ & $\mathbf{L}_{5}$ & $\mathbf{L}_{6}$ \\
\hline 10 & 5 & 0.000 & 0.005 & 0.056 & 0.594 & 0.002 & 0.029 \\
\hline 15 & 10 & 0.003 & 0.000 & 0.053 & 0.034 & 0.048 & 0.003 \\
\hline 20 & 15 & 0.052 & 0.012 & 1. 735 & 0.165 & 0.129 & 0.049 \\
\hline 25 & 20 & 0.000 & 0.033 & 0.079 & 0.658 & 0.003 & 0.098 \\
\hline 30 & 25 & 0.001 & 0.026 & 0.027 & 0.482 & 0.006 & 0.083 \\
\hline 35 & 30 & 0.000 & 0.000 & 0.018 & 0.035 & 0.000 & 0.003 \\
\hline 40 & 35 & 0.000 & 0.004 & 0.017 & 0.014 & 0.004 & 0.021 \\
\hline 45 & 40 & 0.004 & 0.001 & 0.133 & 0.002 & 0.015 & 0.009 \\
\hline 50 & 45 & 0.007 & 0.000 & 0.231 & 0.020 & 0.022 & 0.004 \\
\hline
\end{tabular}

Table 1, shows the Bayes estimarors of $\theta_{1}$ and $\theta_{2}$ under SELF, LLF, GELF, PLF, and Loss functions of them that $L_{1}=L\left(\hat{\theta}_{1 B S}, \theta\right), L_{2}=L\left(\hat{\theta}_{2 B S}, \theta\right)$, $L_{3}=L\left(\hat{\theta}_{1 B L}, \theta\right), L_{4}=L\left(\hat{\theta}_{2 B L}, \theta\right), L_{5}=L\left(\hat{\theta}_{1 B E}, \theta\right), L_{6}=L\left(\hat{\theta}_{2 B E}, \theta\right), L_{7}=L\left(\hat{\theta}_{1 B P}, \theta\right), L_{8}=L\left(\hat{\theta}_{2 B P}, \theta\right)$. 


\section{References}

[1] Srivastata. U. (2012). Bayesian estimation of shift point in Poisson model under asymmetric loss functions, DDU Gorakhpur University;Gorakhpur-273009, U. P,. India. pp $31-42$

[2] Ganji. M . (2010). Bayes estimation of reliability function for a changing exponential family model, University of MohegheghArdabili. world academy of science, engineering and technology 412010.

[3] Aitchison. J and Dunsmore, IR. (1975). Statistical prediction analysis, London, Cambridge University press.

[4] Basu, A. P. and Ebrahimi. N. (1991). Bayesian approach to life testing and reliability estimation using asymmetric loss function. jour. of stat. planning \& inf. (29)(1991)21-31

[5] Berger. J. O. (1985). Statistical decision theory and bayesiananalysis, springerverlag.

[6] Zellner. A. (1986). Bayesian estimation and prediction using asymmetric loss function. Journal of the American statistical Association, 1986. Vol . 81 . No. 394. Theory and Methods.

[7] Broemeling. L. D. (1985). Bayesian analysis of linear models. NewYork;Marcel-Dekker.

[8] Fotopoulos. S. B. and Jandhyala. V. K. (2001). Maximum likelihood estimation of shift point for exponentially distributed random variables. Statist. and Prob.

[9] Calabria. R, Pulcini. G. (1996). Point estimation under asymmetric loss functions for left truncated exponential samples. Commun. State. Theor, Meth. 25(3); 585-600.

[10] Broemeling. L. D. Tsurumi. H. (1987). Econometrics and structural shift. NewYork; Marcel-Dekker.

[11] Boudjellaba. H, MacGibbon. B, andSawyer. P. (2001). On exact inference for change in poissonsequence, communications in statistics A theory and methods, 30(3), 407-434.

[12] Zack. S. (1983). Survey of classical and Bayesian approaches to the shift point problem:fixed sample and sequential procedures for testing and estimation. Recent advances in statistics. Hermanchernoff best shrift. NewYork:academic Press.

[13] Wu. Z, Tian. Y. (2005). Weighted-loss-function CUSUM chart for monitoring mean and variance of production process. Int. J. product. Res. 43:3027-3044.
[14] Parsian. A, Kirmani. S. N. U. A. (2002). Estimation under linex loss function. Hand book of applied econometrics and statistical inference, Statistics text book and monograph. 165. New York:Marcel Dekker, pp. 53-76

[15] Hinkley. D. V. (1970). Inference about the shift point in a sequence of random variables. Biometrica, 57(1), 1-17

[16] Hinkley. D. V, Hinkley. E. A. (1970). Inference about the change point in a sequence of binomial variables. Biometrica, 57 (3), 477-488.

[17] Ohtani. K. (1995). Generalized ridge regression estimators under the linex loss function statist. Pap. 36;99-110.

[18] Shah. J. B, Patel. M. N. (2007). Bayes estimation of shift point in geometric sequence. Commun. Statist. Theor. Meth. 36(6); 1139-1151.

[19] Varian. H. R. (1975). Abayesian approach to real estate assessement. In:Feinger. Z, ed. Studies in Bayesian econometrics and statistics and statistics in Honor of Leonard J. Savage. Amesterdam:North Holland.

[20] Worthley. K. J. (1986). Confidence region and test for shift point in a sequence of exponential family random variables. Biometrica, 73(1), 91-104.

[21] Wael. A. J. (2010) Bayes estimator of one parameter Gamma distribution under quadratic and linex loss fnction. iragi journal of statistical science, (16)2010, pp[13-28].

[22] Javier Giron. F. (2007). Objective Bayesian analysis of multiple change points for linear models. Bayesian statistics 8, pp 1-27.

[23] Srivastava. U. D. D. U. (2012). GorakhpourUniversity. change point estimation on pareto sequence under asymmetric loss functions using Bayesian technique.

[24] Ertefaie. A, Parsian. A. (2005). Bayesian estimation for the pareto income distribution under linex loss function.

[25] Dey. S. (2012). Bayesian estimation of the parameter and reliability function of an Inverse Rayleigh distribution. Malaysian journal of mathematical sciences 6(1): 116124(2012).

[26] Rukhin. A. L. (1991). change point estimation under assymetricloss. NSA grant MD904-93-H-3015.

[27] Parsian. A, AziziSazi . S. (2008). Constrained Bayes Estimators under Balanced Loss Functions. J. of Stat. Sci. 2008; 2 (1) :16-21 